\title{
Impediments in Healthy Ageing: A Short Review
}

\section{Ramya Dwivedi*}

Associate Professor, Department of Psychology, Jain University, Bangalore, India

*Corresponding Author: Ramya Dwivedi, Associate Professor, Department of Psychology, Jain University, Bangalore, India.
Received: August 26, 2021

Published: September 23, 2021

(C) All rights are reserved by Ramya Dwivedi.

\begin{abstract}
In recent years, studies have brought to light preclinical evidence of the ability of cannabinoids to reduce tumor growth in animal models, and clinical trials have been designed to study this activity in patients with glioblastoma. The data in animal models show promising results. So far it has been seen that the administration of THC is safe, and can be carried out without psychoactive effects. These molecules act through receptors coupled to the G protein, which are part of the endocannabinoid system and which have been called CB1 and CB2. THC can attenuate tumor progression in some patients, and appears to have a positive effect on survival, similar to that generated by other chemotherapeutic agents. More trials are needed to validate this antitumor action, both in combination with other therapies, and independently. At this time there is not enough scientific evidence to be able to conclusively affirm that cannabinoid treatment can contribute improve current therapies given to patients with brain tumors or other types of tumors.
\end{abstract}

Keywords: Healthy Ageing; Cognitive Impairments; Challenges of Old Age; Memory; Neuropsychological Changes

\section{Introduction}

Ageing care in India has been a prime concern for most of the gerontologists. Increased life expectancy rates in the elderly population can be contributed to the improved medical and health care amenities. With the rise in elderly population, demand for holistic concern and services also tend to grow. The geriatric survey projects that the proportion of Indians aged 60 years and older will rise from $7.5 \%$ in 2010 to $11.1 \%$ in 2025 . The number of the elderly population in India is expected to reach 158.7 million in 2025, which in 2010 was somewhere around 91.6 million. The demographic profiles of elderly as reflected in the figure 1 also observes an impressive gain in terms of the life expectancy ratio.

For any developing country to progress, geriatric population accounts for a burden on the national resource [2]. The population

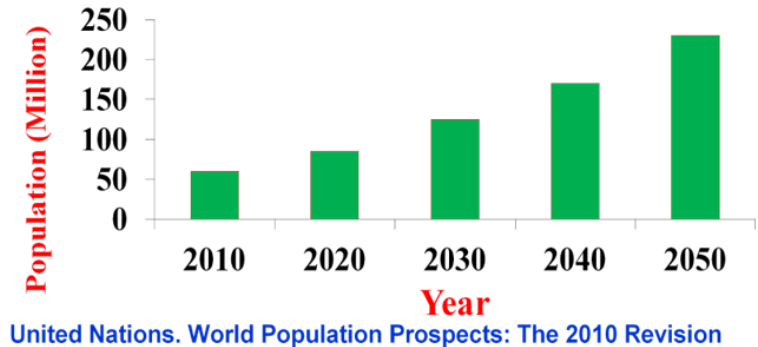

Figure 1: Showing the growth of population aged 65 years and above in India (Reference- United Nations World Population Prospects: The 2010 Revision) [1].

Citation: Ramya Dwivedi. "Impediments in Healthy Ageing: A Short Review". Acta Scientific Neurology 4.10 (2021): 49-52. 
needs both medical as well as social and psychological attention. Old age is also a vulnerable age for physical as well as cognitive deficiencies. Cognitive deficiencies lead to a number of severe forms of degenerative disorders like Alzheimer's disease dementia and Parkinson's disease that may further affect their neurological as well as psychological functioning. A number of psycho- social reasons can be attributed for such dysfunctions. To quote a few would be socio- economic status, cultural norms, stress and its coping strategy, dietary patterns social support, level of education, physical activity etc. Hence it is a task requiring great strength for the policy maker to come up with the factors emerging as critical biomarkers against healthy and successful ageing.

\section{Cognitive ability and ageing}

Ageing can lead to a number of changes in the various domains. Memory functioning alters with a change in crystallized intelligence which is defined as the knowledge about the world and fluid intelligence which is a basic cognitive ability necessary to evaluate and analyze information about a problem or a decision [3]. There can be individual differences operating upon the variations in cognitive ability. The difference gets reflected under two different notions. It either gets reflected with the difference in prior cognitive ability or with the difference in degree to which change has taken place. Literature reviews, however states that there are some areas that are more prone to general decline than the more specific ones. General ageing decline is characterized with impairments in some aspects of memory, executive functions, processing speed and reasoning and slower information processing speed. Cognitive abilities tend to decline with age. However as mentioned earlier, it is important to understand that some of this decline is normal with the ageing process while some decline might reflect atypical changes in the brain. The same is highlighted in the figure below. It depicts the different range of impairments that can be experienced by someone progressing towards cognitive dementia or MCI. Asymptomatic AD would highlight pathological changes in the brain of cognitively normal older adults. People with such symptoms might come up to a clinician with some subjective memory complains that does not detect the presence of Alzheimer's or other forms of dementia.

\section{Role of lifestyle factors on cognitive functioning}

A number of reasons have been attributed towards the impaired cognitive functioning among individuals. Diverse factors play a role in either preventing or promoting the progression of

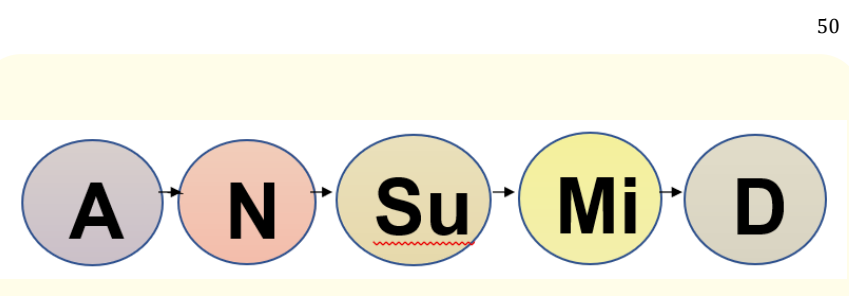

Figure 2: Showing the spectrum of cognitive impairment.

cognitive impairment. Few of these factors have been elucidated below. Research findings suggest the role of diets in predicting the onset of cognitive decline. Current researches focus on the role of specific dietary elements and patterns that may help to delay this onset. Diets rich in Vitamin B, antioxidant and Omega 3 fatty acids are essential for maintaining normal brain function of the memory [4]. Mediterranean diet is also found to have a significant effect in delaying cognitive decline. Mediterranean diet is a diet rich in vegetables, legumes, fruits, nuts cereals fish and moderate amount of alcohol (particularly red wine) and should be low in meat and dairy products. Diets that add on the risk for cognitive decline are the ones rich in refined sugar, cholesterol and trans-fat [5].

Apart from the dietary make up, other factors that may lead to challenges and complexities in healthy ageing can be the other lifestyle factors like physical activity, exercise and cognitive reserve etc. The well-known 'cognitive reserve' hypothesis states that individuals who are more cognitively active or engaged with constructive activities subsequently would have lesser probability to delay the onset of cognitive impairment $[6,7]$.

Elderly in our country are the most vulnerable to develop psychological issues. One reason can be the lack of social security system. The urban elderly still have an option of hiring domestic help to assist them with their activity of daily livings. But this has led to an increase in social isolation and loneliness. By the time an elderly reaches their vulnerable age, their sons and daughters are already married and busy with their own family which narrows down their immediate family circle. An addition to this social aspect is the loss of a spouse that tends to count on the factors making the individual more vulnerable against active ageing. In the same regard, financial dependence also stands to be one of the prime factors affecting the psychological health and functioning. With regard to the financial 
support, pensions and social security services is only limited to the people working in public sector. The elderly population of rural area is less vulnerable to aging issues as compared to the urban inhabitants due to the presence of joint family system. Research studies have found that according to the gender census survey, major proportion of elderly women were poorer and have also recorded the highest negative affective psychological conditions. They were also recorded with least health insurance coverage and recorded the lowest consumption expenditure [8]. In a study it was reported that $47 \%$ of the elderly felt unhappy and $36.2 \%$ reported as they were a liability into the family. The research further reported that half of the respondents felt neglected and sad and reported of experiencing an indifferent approach towards them [9].

Active and engaged lifestyles are also considered beneficial for cognitive ageing. Brains that are actively involved in cognitively stimulating activity tend to protect individuals against cognitive decline. Education also has interplay with cognitive functioning. People who have formal education and belong to higher social class are associated with less cognitive decline $[10,11]$.

\section{Conclusion}

The spectrum of complexities and challenges during old age are multifaceted. One of the prime most challenges towards active and healthy ageing is cognitive decline, which can be defined as a collection of symptoms characterizing difficulties related with retention and recall of memory. It is a natural feature that most of the cognitive functions decline with age. The severity of symptoms depends on the progression of impairment. Thus, there is a need to establish s point where the normal decline ends, and the disease vulnerable progression begins. Cognitive decline does not stand to be the only sole feature against healthy and active ageing. A number of life course factors like social support, level of stress exposure, physical exercise education and dietary intake also have a role in promoting healthy and active ageing [12].

Diets rich in Omega-3 fatty acids, vitamin B-12 and B-6 have shown to be a protective factor against cognitive decline. Similarly the role of cognitively stimulating activity also plays an active role in building cognitive reserves against cognitive decay. Researches have also studied the role of education in predicting sound cognitive health. Advanced level of educational attainment works as a protective factor against any kind of cognitive decline. Last but not the least; social support has emerged as the most significant predictor of physical as well as psychological health in relation to stress. Individuals exposed to adequate levels of social support are less prone to stress inducing situations and are better at building and using coping strategies.

\section{Bibliography}

1. United Nations World Population Prospects: The 2010 Revision.

2. WHO. Tufts University School of Nutrition and Policy. Keep fit for life: Meeting the nutritional needs of older persons. WHO, Geneva, Switzerland (2002).

3. Douglas A Hershey, Helen C. Gutierrez, in Aging and Decision Making (2015).

4. Parrott MD and Greenwood CE. "Dietary influences on cognitive function with aging: from high-fat diets to healthful eating". Annals of the New York Academy of Sciences 1114 (2007): 389-397.

5. Ordovas JM. "Nutrition and cognitive health Foresight Mental Capital and Wellbeing Project". Final Project Report. London The Government Office for Science (2008).

6. Whalley LJ., et al. "Cognitive reserve and the neurobiology of cognitive aging". Ageing Research Reviews 3 (2004): 369-382.

7. Foresight Mental Capital and Wellbeing Project Final Project Report. London The Government Office for Science (2008).

8. Hiremath SS. "The Health Status of Rural Elderly Women in India: A Case Study". International Journal of Criminology and Sociological Theory 5 (2012): 960-963.

9. Lena A., et al. "Health and Socio Problems of the Elderly: A Cross Sectional Study in Udupi taluk, Karnataka". Indian Journal of Community Medicine 34 (2009): 131-134.

10. Ian J., et al. "Starr, Age-associated cognitive decline". British Medical Bulletin 92.1 (2009): 135-152.

11. Mane AB. "Ageing in India: Some Social Challenges to Elderly Care". Journal of Gerontology and Geriatric Research 5.2 (2016).

12. Archana Singh-Manoux and Mika Kivimäki. "The importance of cognitive ageing for understanding dementia". Journal of the American Ageing Association 32.4 (2010): 509-512. 
13. Daniel L Murman. "The Impact of Age on Cognition". Seminars in Hearing 36.3 (2015): 111-121.

14. Rakesh Kumar Tripathi and S C Tiwari. "Cognitive Dysfunction in Normally Aging Urban Older Adults: A Community-based Study". Indian Journal of Psychological Medicine 33.2 (2011): 177-181.

Volume 4 Issue 10 October 2021

(C) All rights are reserved by Ramya Dwivedi. 